OPEN ACCESS

Edited by:

Rafael Christophe Freire,

Queen's University, Canada

Reviewed by:

Yasuhiro Kaneda,

Iwaki Clinic, Japan

Mohammed Al Alawi,

Sultan Qaboos University, Oman

*Correspondence:

Yanbin Jia

yanbinjia2006@163.com

†These authors have contributed equally to this work

Specialty section:

This article was submitted to Mood and Anxiety Disorders,

a section of the journal

Frontiers in Psychiatry

Received: 21 May 2020 Accepted: 19 January 2021

Published: 03 March 2021

Citation:

Liao X, Lai S, Zhong S, Wang Y, Zhang $Y$, Shen S, Huang $H$, Chen $G$, Chen $F$ and Jia $Y$ (2021) Interaction of Serum Copper and Neurometabolites

on Executive Dysfunction in Unmedicated Patients With Major Depressive Disorder.

Front. Psychiatry 12:564375

doi: 10.3389/fpsyt.2021.564375

\section{Interaction of Serum Copper and Neurometabolites on Executive Dysfunction in Unmedicated Patients With Major Depressive Disorder}

\author{
Xiaoxiao Liao ${ }^{1,2 t}$, Shunkai Lai ${ }^{1+}$, Shuming Zhong ${ }^{1 \dagger}$, Ying Wang ${ }^{3}$, Yiliang Zhang ${ }^{1}$, \\ Shiyi Shen ${ }^{4}$, Hui Huang ${ }^{4}$, Guanmao Chen ${ }^{3}$, Feng Chen ${ }^{3}$ and Yanbin Jia ${ }^{\text {* }}$ \\ ${ }^{1}$ Department of Psychiatry, First Affiliated Hospital of Jinan University, Guangzhou, China, ${ }^{2}$ Jiangmen Central Hospital, \\ Jiangmen, China, ${ }^{3}$ Medical Imaging Center, First Affiliated Hospital of Jinan University, Guangzhou, China, ${ }^{4}$ School of \\ Management, Jinan University, Guangzhou, China
}

Objective: The mechanism of executive function (EF) impairment in major depressive disorder (MDD) remains unclear. Previous studies have demonstrated that altered serum copper levels and neurometabolic alterations may be associated with the psychopathology and cognitive impairment of MDD. While, their inter-relationships in MDD remain uncertain. The present study aims to assess whether the interaction between serum copper levels and neurometabolic alterations is involved in the deficit of executive function (EF) in patients with unmedicated MDD.

Methods: Serum copper levels and EFs were measured in 41 MDD patients and 50 control subjects. EFs were evaluated by Trail Making Test, Part-B (TMT-B), Digit Symbol Substitution Test (DSST), Wisconsin Card Sorting Task (WCST), and Semantic Verbal Fluency testing (SVFT). Additionally, 41 patients and 41 healthy controls underwent proton magnetic resonance spectroscopy $\left({ }^{1} \mathrm{H}-\mathrm{MRS}\right)$ to obtain ratios of $\mathrm{N}$-acetyl aspartate to creatine (NAA/Cr) and choline-containing compounds to creatine ( $\mathrm{Cho} / \mathrm{Cr}$ ) in the lenticular nucleus (LN) of basal ganglia (BG). Finally, association and interaction analysis were conducted to investigate their inter-relationships.

Results: The results showed that patients performed worse in the DSST, WCST, TMT-B time and SVFT. Moreover, patients had higher serum copper levels, but lower NAA/Cr ratios in left $L N$ of $B G$ than healthy controls. In patients, serum copper levels were found to significantly negative associated with Categories Completed (CC) number of WCST ( $r=-0.408, p=0.008$ ), and positive associated with the Total Errors (TE) and Nonperseverative Errors (PE) number of WCST $(r=0.356, p=0.023 ; r=-0.356$, $p=0.022)$. In addition, the NAA/Cr ratios of left $L N$ were found to significantly negative associated with VFS ( $r=-0.401, p=0.009$ ), as well as negative associated with serum copper levels $(r=-0.365, p=0.019)$. Finally, the interaction between copper and NAA may as influencing factors for SVFT and CC number of WCST in patients.

Conclusion: Our results indicated that the interaction of abnormal copper levels and NAA/Cr neurometabolic disruption of the LN may impact executive dysfunction, and this may relevant to the pathophysiology of executive impairment in MDD patients.

Keywords: major depressive disorder, copper, executive function, lenticular nucleus, proton magnetic resonance spectroscopy 


\section{INTRODUCTION}

In China, the estimation of overall current, 12-month, and lifetime prevalence of Major depressive disorder (MDD) is 1.3$2.7 \%, 2.3 \%$, and $2.8-3.3 \%$ (1). MDD is associated with cognitive cognitive deficits and it is established that depression increases the risk of cognitive impairment, particularly on executive functions (EF) such as memory, attention, and problem-solving $(2,3)$. Impairment of EF is widely recognized as a primary characteristic of MDD. Numerous studies have demonstrated that adults with MDD have mild to moderate EF deficits affecting inhibitory control, cognitive flexibility, and verbal fluency (46). Recent studies showed that $20-30 \%$ of individuals with MDD have pronounced executive dysfunction (7) persisting into the euthymic or more stable phases $(3,8)$. Furthermore, premorbid EF deficit is considered a trait-marker for adult MDD (9), and may prevent MDD patients from full remission (10). Nevertheless, the exact physiological mechanisms linking MDD with cognitive impairment are poorly known.

Trace elements play a role in the pathogenesis of MDD. Indeed, copper and zinc play important roles in MDD (11). Illustrating this relationship, Wilson's disease, or hepatolenticular degeneration, is a rare inherited autosomal recessive disorder of copper metabolism leading to copper accumulation in the liver and extrahepatic organs such as the lenticular nucleus and cornea (12). Among patients with Wilson's disease, up to 30 and $25 \%$ have symptoms of depression and cognitive impairment, respectively, and 25\% patients are ultimately diagnosed with depression (13). Metabolic disturbance of copper is observed in patients with MDD, with higher serum copper levels (14). Inconsistently, previous studies examined the serum copper levels of patients with MDD and found lower or unchanged levels in patients with MDD (15). It is hypothesized that high serum copper is associated with higher oxidative stress, which plays physiological roles in cellular signaling and can cause cell damage and increased glutamate levels (16). Reactive oxygen species are associated with neurodegenerative processes and depression (17), and cognitive dysfunction $(18,19)$. While the underlying relationship between copper disturbance and executive function impairments in patients with MDD remain uncertain.

Imaging of patients with MDD reveals structural and functional abnormalities in the prefrontal cortex, anterior cingulate cortex, amygdala, hippocampus, basal ganglia and thalamus (20-22). As deep gray matter structures of the brain, the basal ganglia was critically implicated in the modulation of a wide range of motor, complex cognitive, and emotional processing as well as behavioral function $(23,24)$. Dysfunction of the lenticular nuclei, which are part of the basal ganglia, is observed in patients with MDD (25). Critical brain regions of adults with MDD house neurometabolic abnormalities that may reveal the biological mechanisms underlying the pathophysiology of their disorder $(26,27)$. Findings from neuropsychological and proton magnetic resonance spectroscopy $\left({ }^{1} \mathrm{H}\right.$-MRS $)$ studies support the important role of the basal ganglia in EF (28). Yet no significant differences in NAA/Cr or Cho/Cr ratios of the bilateral lenticular nucleus of the basal ganglia are evident in adults with MDD or in healthy controls (25). Likewise, a meta-analysis also finds no alteration of
NAA in the basal ganglia of adults with MDD (29). Nevertheless, one study indicates that adults with MDD had a significantly lower $\mathrm{NAA} / \mathrm{Cr}$ in the right caudate and a significantly higher mean $\mathrm{Cho} / \mathrm{Cr}$ in the right putamen of basal ganglia (30). Higher choline and creatine concentrations are also found in the basal ganglia of adolescents with MDD (31). Indeed, basal ganglia have a role in disinhibition and planning and are partially involved in the regulation of emotion and cognitive functions.

Neurometabolic changes in critical brain regions are closely related to cognitive dysfunction in MDD. Lower NAA in the prefrontal cortex positively correlates with memory impairment in both adult and adolescent depression (25, 32). Altered NAA concentrations within the thalamus also parallel working memory impairment $(33,34)$. Yet an unchanged NAA/Cr ratio in the lenticular nucleus bears no relationship to working memory impairment related to MDD (25). Nonetheless, a lower $\mathrm{NAA} / \mathrm{Cr}$ ratio in the left lenticular nucleus of the basal ganglia corresponds clearly to executive dysfunction in adults with bipolar II depression $(28,35)$. Furthermore, copper disturbance also have a close relationship with brain metabolic activity of the mood disorders. Previous studies have shown that increased copper levels were involved in the dysfunction of serotoninergic and dopaminergic metabolism in the LN of anxiety (36). Our recent study demonstrated that increased copper levels were negatively correlated with $\mathrm{NAA} / \mathrm{Cr}$ ratios in the right $\mathrm{LN}$ of patients with MDD (37). But how copper disturbance interacts with the neurometabolic of the LN to result in cognitive impairment in patients with MDD begs investigation. Therefore, unique studies are needed to explore the interaction of abnormal copper levels and neurometabolic disruption may impact EF impairment in patients with MDD.

In consideration of this knowledge gap, we conducted the current study, which utilizes 3.0 Tesla ${ }^{1} \mathrm{H}$-MRS and assessments of neuropsychological function to explore the underlying relationship between neurometabolic change, copper disturbance and executive function in unmedicated patients with MDD. The metabolites NAA, Cho, and Cr were detected in vivo, with NAA and Cho reported as a ratio to $\mathrm{Cr}$, since $\mathrm{Cr}$ level is relatively stable within the brain. Executive function was assessed by the Wisconsin Card Sorting Task (WCST), Trail Making Test Part-B (TMT-B), and Digit Symbol Substitution Test (DSST). We sought to find the interrelationships between copper levels, executive function, and neurometabolic alterations of LN on MRS in unmedicated patients with MDD.

\section{METHODS}

\section{Study Design}

The present study was a cross-sectional design. We recruited unmedicated patients with MDD, as well as a comparable number of volunteers participated as healthy controls to analysis the difference and interrelationship of neurometabolic ratios, copper levels and executive function.

\section{Participants}

Patients with MDD were recruited from the psychiatry department of the First Affiliated Hospital of Jinan University 
(Guangzhou, China) between October 2014 and April 2017. The age of the participants was restricted to 17-55 years to minimize the interference of aging and vascular diseases. Two experienced psychiatrists diagnosed each participant using the Structured Clinical Interview for Diagnostic and Statistical Manual of Mental Disorders (4 Edition) (DSM-IV) criteria Patient Edition. All patients were with scores of $>20$ on the 24 item Hamilton Depression Rating Scale (HDRS), and the scores of Young Manic Rating Scale (YMRS) were $<6$. The exclusion criteria were: (1) presence of other psychiatric disorders (bipolar disorder, schizophrenic episode, or panic attack) and symptoms; (2) history of any psychotropic medication, psychotherapy, or electroconvulsive therapy; (3) history of neurological or organic brain disorder; (4) alcohol/substance abuse within 6 months before study entry; (5) any physical illness demonstrated by personal history, or clinical or laboratory examinations; or (6) first-degree relatives having a history of neurological or mental illness.

A comparable number of volunteers participated as healthy controls were recruited via local advertisements. They were carefully screened through a diagnostic interview and the Structured Clinical Interview for DSM-IV Non-patient Edition (SCID-NP) to rule out the presence of current or past psychiatric illness. Additional exclusion criteria for controls were any history of psychiatric illness in first-degree relatives, and current or past significant medical or neurological illness.

All participants were right-handed and were scanned within $48 \mathrm{~h}$ of initial contact. The study was approved by the Ethics Committee of the First Affiliated Hospital of Jinan University, China. All subjects signed a written informed consent form after a full written and verbal explanation of the study.

\section{Executive Function (EF) Assessment}

In total, 41 patients with unmedicated MDD and 50 healthy controls underwent cognitive assessment by two trained graduated students who were blinded to the diagnosis of each participant. Executive function assessment consisted of three tasks: Trail Making Test, Part-B (TMT-B), Digit Symbol Substitution Test (DSST), Wisconsin Card Sorting Task (WCST), and Semantic Verbal Fluency testing (SVFT).

\section{Trace Element Testing}

In total, 41 patients with unmedicated MDD and 41 healthy controls provided blood for trace element detection. A 5-mL fasting venous blood sample was obtained at 7:00-8:00 A.M. by routine venipuncture from all participants. Blood samples were sent to a centralized laboratory that performed analysis following standard procedures. All blood samples were centrifuged and serum samples were obtained. The serum samples were kept at $-20^{\circ} \mathrm{C}$. Atomic absorption spectroscopy (AAS) was used to determine the levels of serum copper.

\section{Image Acquisition}

In total, 41 patients with unmedicated MDD and 41 healthy controls underwent MRI scanning. ${ }^{1} \mathrm{H}$-MRS was conducted under resting conditions. Both magnetic resonance imaging (MRI) and ${ }^{1} \mathrm{H}$-MRS were performed on a clinical $3.0 \mathrm{~T}$ GE
Discovery MR system (General Electric, Milwaukee, WI, USA) with a conventional gradient system and a standard eightchannel head coil. The subjects were lying in the supine position and the nasion served as a landmark. Ear plugs and foam pads were used to reduce noise and minimize head motion. Routine axial $\mathrm{T}_{1}$-weighted fluid attenuation inversion recovery $\left(\mathrm{T}_{1}\right.$ Flair) [repetition time $(\mathrm{TR})=1,000 \mathrm{~ms}$, echo-time $(\mathrm{TE})=144 \mathrm{~ms}$ ] and fast spin echo $\mathrm{T}_{2}$-weighted $\mathrm{MR}$ images $(\mathrm{TR}=3,500 \mathrm{~ms}$, $\mathrm{TE}=$ $102 \mathrm{~ms}$ ) were obtained to confirm the absence of any structural and signal abnormality of the brain.

All spectra were acquired using a $2 \mathrm{D}$ multi-voxel technique. Axial $\mathrm{T}_{2}$-weighted $\mathrm{MR}$ images were used for anatomic localization $(\mathrm{TR}=3,500 \mathrm{~ms}, \mathrm{TE}=102 \mathrm{~ms}$, slice thickness $=5 \mathrm{~mm}$, without gap). For ${ }^{1} \mathrm{H}$-MRS, the positions of the volumes of interest (VOIs) were located in the bilateral LN showed in Figure 1A (large white box). The size of the VOI was 50 nominal voxels $(7.5 \times 7.5 \times 10 \mathrm{~mm})$. Single-section $2 \mathrm{D}$ multivoxel ${ }^{1} \mathrm{H}$-MRS was acquired using a point resolved spectroscopy sequence (PRESS) with water suppression by a chemical shift selective saturation (CHESS) pulse. The acquisition parameters were: $\mathrm{TR}=1,000 \mathrm{~ms}$; $\mathrm{TE}=144 \mathrm{~ms}$; numbers of excitation $=$ 1 ; spatial matrix $=256 \times 256$; field of view $=180 \times 180 \mathrm{~mm}$; slice thickness $=10 \mathrm{~mm}$; and nominal voxel size of $7.5 \times 7.5$ $\times 10 \mathrm{~mm}$. Additional saturation bands were placed outside the VOI to minimize lipid contamination from the scalp. Automatic prescanning was performed before each spectroscopic scan to achieve an optimal full width half maximum of $10 \mathrm{~Hz}$. Total acquisition time for the ${ }^{1} \mathrm{H}$-MRS sequence was $5 \mathrm{~min}$ and $28 \mathrm{~s}$.

The analyses of the spectral dataset were performed with the manufacturer-supplied software package program of the MR system (Sun, Advantage windows ADW4.5, General Electric, Milwaukee, WI, USA). Voxels were repositioned in the left and right LN. Each individual VOI was composed of four voxels (1 $\times 1 \times 1 \mathrm{~mm}$ ). The final measurement value of the individual VOI was the average value of the measurement value of four voxels. Each spectrum was evaluated for the peak area of Cho at $3.22 \mathrm{ppm}, \mathrm{Cr}$ at $3.03 \mathrm{ppm}$, and NAA at $2.02 \mathrm{ppm}$ (showed in Figure 1B). A trained radiologist who was blinded to the diagnosis of each participant carried out voxel placement for spectroscopy. The values of the NAA/Cr and $\mathrm{Cho} / \mathrm{Cr}$ ratios were used for analyses.

\section{Statistical Analysis}

Data analysis was performed using SPSS 24.0 (SPSS Inc., Chicago, IL, USA). Two-tailed significance level was set at $p<0.05$. All indicators (demographics, clinical data, executive function, metabolic data, and the levels of serum copper) were assessed for normal distribution using the Kolmogorov-Smirnov test and using the Levene's test for equality of error variances.

When comparing group differences in terms of demographic and clinical data, the Student $t$ test was used if distribution was normal and the Mann-Whitney $U$ test was used if distribution was skewed. The chi-square test was performed to compare the differences in sex between MDD and controls. 
A

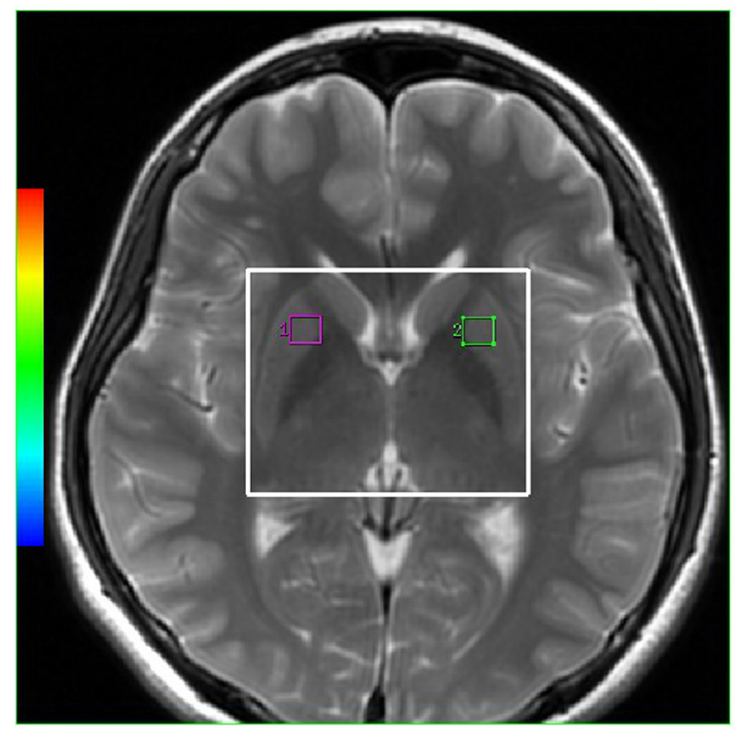

B

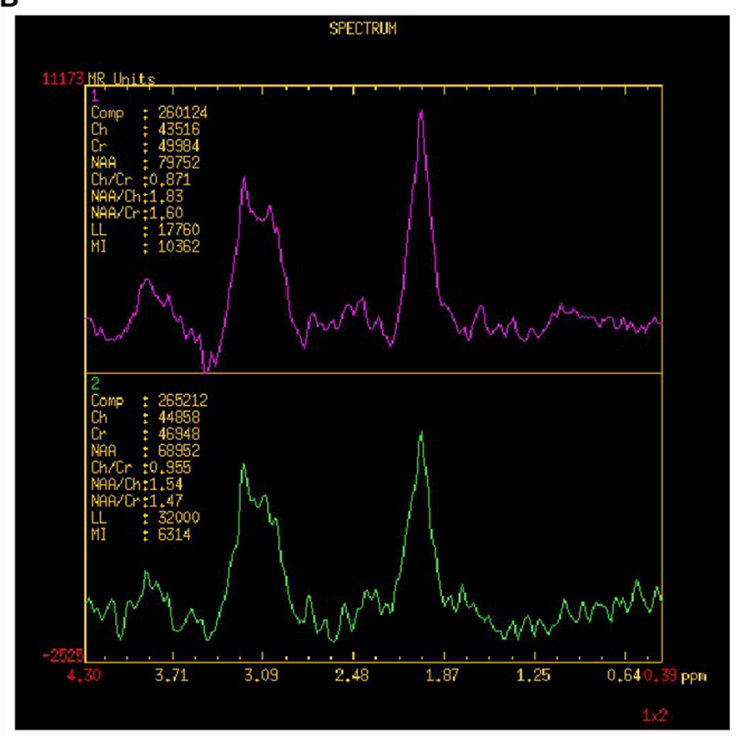

FIGURE 1 | (A,B) Shows the VOI location in an MRI scan of the brain. Single section 2D multi-voxel $1 \mathrm{H}-\mathrm{MRS}$ was acquired using a point resolved spectroscopy sequence (PRESS) with water suppression by a chemical shift selective saturation (CHESS) pulse. Magnetic resonance image (MRI) scan of healthy control subject showing location of magnetic resonance spectroscopy (MRS) of volume of interest (VOI) placed in the lenticular nucleus (LN) of basal ganglia (BG). The large white box represents the VOlx for MRS acquisition, and small white boxes depict the individual VOls for spectral analysis. NAA, $\mathrm{N}$-acetylaspartate; Cho, choline; ml, myo-Inositol; Cr, creatine.

Second, Student $t$ test and the Mann-Whitney $U$ test was used to test the differences in biochemical metabolism ratios, serum copper levels and executive function. A Bonferroni post hoc test was applied to correct for multiple comparisons, with the threshold for significance was set to $p<0.004$ (adjusted $\alpha$ $=0.004,0.05 / 12$ ).
Third, we performed Pearson correlation analyses to establish altered serum copper levels, brain biochemical metabolite ratios, and executive performance for patients with MDD.

Finally, multiple linear regression analyses were performed in $\mathrm{R}$ version 3.3.1. and adopted to model the relationship between serum copper levels, brain neurometabolite ratios, and executive performance. We set the significance levels at $p<0.05$.

\section{RESULTS}

\section{Demographics and Cognition of Unmedicated MDD Patients and Healthy Controls}

Demographic, clinical data, and executive function performance for MDD patients and healthy controls were listed in Table 1. There were no significant differences in sex, and age between the two groups $(p>0.05)$. Patients with MDD had a significantly lower score of DSCS, the CC number of WCST and SVFT, and significantly higher in the TE, PE number of WCST and TMTB time compared with controls. Those differences of DSST, PE number of WCST, TMT-B time and SVFT were still exhibit after Bonferroni correction at $p<0.004$.

\section{Serum Copper Levels and Neurometabolism in MDD Patients and Healthy Controls}

The MDD group showed significantly higher serum copper levels compared to HCs $(t=3.745, p<0.001)$ (Table 2). Patients with MDD had a significantly lower NAA/Cr ratio in the left LN compared to controls $(z=-2.808, p=0.006)$, but this results failed to exhibit any significant difference in the Bonferroni corrections at $p<0.004$.

\section{Correlation Between Abnormal Serum Copper, Cognition and Neurometabolism in MDD}

In the MDD group, serum copper levels were found to significantly negative associated with CC number of WCST ( $r=$ $-0.408, p=0.008)$, and significantly positive associated with the TE and PE number of WCST $(r=0.356, p=0.023 ; r=-0.356, p$ $=0.022$ ) (Table 3). In addition, the NAA/Cr ratios of left LN were found to significantly negative associated with VFS $(r=-0.401$, $p=0.009)$, as well as negative associated with serum copper levels $(r=-0.365, p=0.019)$.

\section{Interaction of Abnormal Serum Copper Levels and Neurometabolic Ratios With Cognitive Function in Patients With MDD}

Multiple regression analyses demonstrated that the $\mathrm{Cu}^{2+} \times \mathrm{NAA}$ interactions in the left $\mathrm{LN}$ was independent predictors for the SVFT $(t=-0.736, p=0.04)$ and WCST CC $(t=-1.482, p=$ $0.02)$ in patients with MDD. 
TABLE 1 | Comparison of clinical and demographic and EF performance between patients with MDD and healthy controls.

\begin{tabular}{|c|c|c|c|c|}
\hline & MDD & HCs & $\chi^{2} / t / Z$ & $p$ \\
\hline Number of subjects & 41 & 50 & - & - \\
\hline Sex (male/female) & $15 / 26$ & $23 / 27$ & 0.821 & $0.365^{a}$ \\
\hline Number of episodes & $1.66 \pm 1.05$ & NA & NA & NA \\
\hline Education(years) & $12.24 \pm 3.34$ & $15.40 \pm 1.99$ & -4.417 & $<0.001^{b^{* \alpha+\alpha}}$ \\
\hline Y-MRS score & $0-6$ & $0-6$ & NA & NA \\
\hline DSST & $55.85 \pm 12.76$ & $69.02 \pm 10.41$ & -5.216 & $<0.001^{b^{*+*}}$ \\
\hline CC,WCST & $5.73 \pm 1.58$ & $6.52 \pm 1.04$ & -248 & $0.025^{\mathrm{b}^{*}}$ \\
\hline TE,WCST & $10.24 \pm 9.55$ & $5.18 \pm 4.28$ & -2.686 & $0.007^{b^{*+}}$ \\
\hline PE,WCST & $6.15 \pm 7.38$ & $2.18 \pm 2.59$ & -3.220 & $0.001^{b+x}$ \\
\hline
\end{tabular}

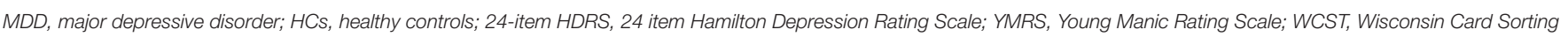

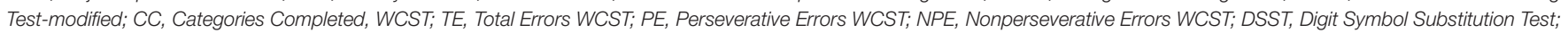
SVFT, Semantic Verbal Fluency testing.

${ }^{a} \chi^{2}$ test; ${ }^{b}$ Mann-Whitney $U$ test; ${ }^{c}$ t-test.

${ }^{*} p<0.05,{ }^{* *} p<0.01,{ }^{* \star *} p<0.001$.

TABLE 2 | Comparison of serum copper levels and neurometabolic characteristics between patients with MDD and healthy controls.

\begin{tabular}{|c|c|c|c|c|}
\hline & MDD & HCs & $\chi^{2} / t / Z$ & $p$ \\
\hline Number of subjects & 41 & 41 & - & - \\
\hline Sex (male/female) & $15 / 26$ & $20 / 21$ & 1.246 & $0.264^{\mathrm{a}}$ \\
\hline Number of episodes & $1.66 \pm 1.05$ & NA & NA & NA \\
\hline Duration of illness (month) & $46.08 \pm 56.39$ & NA & NA & NA \\
\hline Right LN Cho/Cr & $0.77 \pm 0.29$ & $0.89 \pm 0.63$ & -1.415 & $0.157^{b}$ \\
\hline Left LN Cho/Cr & $0.76 \pm 0.17$ & $0.89 \pm 0.57$ & -0.343 & $0.731^{b}$ \\
\hline Right LN NAA/Cr & $1.66 \pm 0.0 .44$ & $1.69 \pm 0.25$ & -1.651 & $0.099^{b}$ \\
\hline Left LN NAA/Cr & $1.52 \pm 0.22$ & $1.69 \pm 0.32$ & -2.808 & $0.006^{b^{\mathrm{b*}}}$ \\
\hline serum copper levels & $16.90 \pm 4.72$ & $13.69 \pm 2.79$ & 3.745 & $<0.001^{\mathrm{C}^{\star \star \star}}$ \\
\hline
\end{tabular}

MDD, major depressive disorder; HCS, healthy controls; 24-item HDRS, 24 item Hamilton Depression Rating Scale; LN, lenticular nucleus of basal ganglia; YMRS, Young Manic Rating Scale.

${ }^{a} \chi^{2}$ test; ${ }^{b}$ Mann-Whitney $U$ test; ${ }^{c} t$-test.

${ }^{* \star} p<0.01,{ }^{* \star *} p<0.001$.

\section{DISCUSSION}

The purpose of our study has been to explore the underlying copper-brain interactions in patients with MDD compared with healthy controls. Ultimately, we found that (1) significant associations between abnormal serum copper levels and executive function deficits in patients with MDD, showing a significantly negative correlation of serum copper levels to CC number of WCST, along with a significantly positive correlation of copper to TE and PE number of WCST; (2) abnormal serum copper levels were also associated with neurometabolic disruption of patients with MDD, showing that copper levels had a significantly inverse association with NAA/Cr ratios in the left LN; and, (3) the interaction of abnormal copper levels and $\mathrm{NAA} / \mathrm{Cr}$ neurometabolic disruption of the $\mathrm{LN}$ was independent predictors for the SVFT and WCST CC in patients with MDD.

Abnormal serum copper is a potential biomarker of MDD $(13,14)$. In our present study, we found significantly higher serum copper levels of patients with MDD when compared with healthy controls, findings consistent with previous studies $(38,39)$. The deposition of copper result in neuronal cell and gliocyte swelling and cystic degeneration, death, and loss, which 
TABLE 3 | Correlation between abnormal serum copper, cognition and neurometabolism in MDD

\begin{tabular}{lcc}
\hline & Left LN NAA/Cr & Serum copper levels \\
\hline DSST & -0.113 & 0.227 \\
CC,WCST & 0.177 & $-0.408^{\star \star}$ \\
TE,WCST & -0.221 & $0.356^{\star}$ \\
PE,WCST & -0.209 & $0.356^{*}$ \\
TMT-B Time & 0.041 & -0.071 \\
SVFT & $-0.401^{\star *}$ & 0.263 \\
Left LN NAA/Cr & NA & $-0.365^{\star}$ \\
serum copper levels & $-0.365^{\star}$ & $\mathrm{NA}$ \\
\hline
\end{tabular}

${ }^{*} p<0.05,{ }^{* *} p<0.01$.

WCST, Wisconsin Card Sorting Test-modified; CC, Categories Completed; WCST; TE, Total Errors WCST; PE, Perseverative Errors WCST; NPE, Nonperseverative Errors WCST; DSST, Digit Symbol Substitution Test; SVFT, Semantic Verbal Fluency testing; LN, lenticular nucleus of basal ganglia.

induce persistent neurological deficits and cognitive dysfunction (40). In the present study, we found that patients with MDD had a significantly lower score of DSST, the CC number of WCST and Verbal fluency, and significantly higher in the TE, PE number of WCST and TMT-B time compared with controls. These results suggest that patients with MDD showed executive dysfunction, consisting with previous research findings (41). Correlation analysis shown that altered serum copper levels were found to significantly associated with CC, TE and PE number of WCST, suggesting a positive relationship between the higher serum copper levels and cognitive impairment risk. A meta-analysis demonstrated increased serum copper concentrations of $\mathrm{AD}$ patients when compared with healthy controls (42). Similarly, a previous study also reported that women with a high concentration of copper in plasma had poorer cognitive function than women with low plasma copper concentration (43). The alteration of $\mathrm{Cu}^{2+}$ homeostasis plays an essential role in the synthesis and functioning of BDNF (44). Copper regulates matrix metalloproteinases and tyrosine phosphatase activity, which promotes the maturation of proBDNF to $\operatorname{BDNF}(45,46)$, and induces the oxidative stress damage (47). Moreover, a recent study indicated that the interaction between decreased BDNF and oxidative damage is involved in the disruption of executive function (48). In alignment with our findings, these results corroborates the notion that copper is essential for the synthesis of neurotransmitters and resultant impairment in cognitive function.

In our present study, we found MDD patients had a significantly lower $\mathrm{NAA} / \mathrm{Cr}$ ratio in the left $\mathrm{LN}$ compared to controls, findings consistent with previous studies (30). NAA is synthesized in neuronal mitochondria and reflects the functional state of neurons. Reduced NAA levels may reflect a loss of neurons or may be a marker of neuronal damage and mitochondrial dysfunction $(49,50)$. Further, we found that the higher copper levels had a significantly inverse association with NAA/Cr ratios in the left LN of patients with MDD. Studies have suggested that the cuprizone (CPZ, a copper chelator) may damage oligodendrocytes by causing demyelinating insult and leading to the appearance of abnormal mental functions, such as schizophrenia symptoms (51). Rats on the CPZ diet showed decreased expression of mRNA transcripts encoding oligodendroglia proteins and displayed a specific deficit in the ability to shift between perceptual dimensions in the attentional set-shifting task, similar to WCST deficits observed in schizophrenia (52). And we did find an significantly negative association between decreased NAA/Cr ratios of left LN and executive dysfunction in patients with MDD. Although the associations cannot provide for causal pathophysiology, it has been suggested that abnormal serum copper levels may impair LN microstructural and eventually generate cognitive impairment.

Furthermore, we also found that the $\mathrm{Cu} \times$ NAA interaction in the left LN was associated with poor executive performance in patients with MDD. Such changes may suggested that abnormal serum copper levels may impair LN microstructural and cause cognitive dysfunction. Here we are able to connect the neurometabolic abnormalities to altered serum copper levels through glutamate system. Firstly, the glutamatergic system also plays an important role in the pathophysiology of MDD (53). And the altered NAA may have a close relationship to and complex interaction with increased glutamate levels (54). Meanwhile, free copper excess alters their cortical glutamatergic neurotransmission (55). Recent studies have shown that copper plays an important role in the regulation of the NMDAR, the $\alpha-$ amino-3-hydroxy-5-methyl-4isoxazole-propionic acid receptor (AMPAR), synaptogenesis and learning and memory function $(56,57)$. In our recent study, we found that the NMDA receptor antagonist can suppress serum copper levels and improving symptoms of anhedonia and anxiety and the cognitive deficits associated with depression (58). Furthermore, copper is involved in oxidative stress processes and immunological system regulation (59). Depression is accompanied by the activation of oxidative stress and inflammatory reaction. And the increased inflammation in major depression may lead to increased glutamate in the basal ganglia in association with glial dysfunction (60), and thus impaired executive function (61). Taken together, these data indicate that the disruption of NAA/Cr neurometabolic of LN and serum copper levels is relevant to the pathophysiology of executive impairment in MDD patients.

\section{LIMITATIONS}

We acknowledge that our study was limited in several ways. Namely, the sample size was relatively small and the participants were highly selected since the study was not a cross-sectional design. Furthermore, other factors may influence executive function: evidence also implicates childhood trauma and early life adversity as risk factors for cognitive deficits (62). Additionally, BDNF, oxidative stress or inflammatory and the levels of glutamate nor $\gamma$-aminobutyrate were not detected that might impact NAA levels over time, which could contribute to brain network and cognitive dysfunction. Further research incorporating biological and psychosocial effects, as well as 
longitudinal research may provide a more complete picture of the relationship between serum copper levels, neurometabolic changes and executive dysfunction.

\section{CONCLUSIONS}

In conclusion, we conducted a correlation analysis on the relationship between serum copper, executive function, and neurometabolites of the LN in unmedicated patients with MDD. Our results indicate that patients with MDD show deficits in executive function and higher serum copper levels, in addition to a significantly negative correlation of serum copper levels and poorer executive performance. Patients with MDD also showed significantly lower NAA/Cr ratios in LN compared to healthy controls. Meanwhile, the abnormal serum copper levels were also associated with decreased NAA/Cr ratios in the left LN. Further, our results indicated that the interaction of abnormal copper levels and NAA/Cr neurometabolic disruption of the LN may impact executive dysfunction in patients with MDD. Despite the previously described limitations, we believe this may relevant to the pathophysiology of executive impairment in MDD patients.

\section{DATA AVAILABILITY STATEMENT}

The raw data supporting the conclusions of this article will be made available by the authors, without undue reservation.

\section{ETHICS STATEMENT}

The studies involving human participants were reviewed and approved by the Ethics Committee of the First Affiliated

\section{REFERENCES}

1. Huang Y, Wang Y, Wang H, Liu Z, Yu X, Yan J, et al. Prevalence of mental disorders in China: a cross-sectional epidemiological study. Lancet Psychiatry. (2019) 6:211-24. doi: 10.1016/S2215-0366(18)30511-X

2. Bortolato B, Carvalho AF, McIntyre RS. Cognitive dysfunction in major depressive disorder: a state-of-the-art clinical review. CNS Neurol Disord Drug Targets. (2014) 13:1804-18. doi: 10.2174/1871527313666141130203823

3. Hasselbalch BJ, Knorr U, Kessing LV. Cognitive impairment in the remitted state of unipolar depressive disorder: a systematic review. J Affect Disord. (2011) 134:20-31. doi: 10.1016/j.jad.2010.11.011

4. Ahern E, Semkovska M. Cognitive functioning in the first-episode of major depressive disorder: a systematic review and meta-analysis. Neuropsychology. (2017) 31:52-72. doi: 10.1037/neu0000319

5. Rock PL, Roiser JP, Riedel WJ, Blackwell AD. Cognitive impairment in depression: a systematic review and meta-analysis. Psychol Med. (2014) 44:2029-40. doi: 10.1017/S0033291713002535

6. Snyder HR. Major depressive disorder is associated with broad impairments on neuropsychological measures of executive function: a meta-analysis and review. Psychol Bull. (2013) 139:81-132. doi: 10.1037/a0028727

7. McIntyre RS, Cha DS, Soczynska JK, Woldeyohannes HO, Gallaugher LA, Kudlow P, et al. Cognitive deficits and functional outcomes in major depressive disorder: determinants, substrates, and treatment interventions. Depress Anxiety. (2013) 30:515-27. doi: 10.1002/da.22063
Hospital of Jinan University, China. All subjects signed a written informed consent form after a full written and verbal explanation of the study. The patients/participants provided their written informed consent to participate in this study.

\section{AUTHOR CONTRIBUTIONS}

YJ, XL, SZ, and SL were involved in the design of this study and contributed to the writing of the manuscript. GC and FC performed the MR scans. GC, FC, and YW performed the MR data analyses. YJ and SZ were involved in diagnosing subjects. SL, SZ, and Yan Xia carried out the statistical analyses. YZ, SS, and $\mathrm{HH}$ were involved in clinical data collection. XL, SL, and SZ wrote the first draft of the manuscript. YJ provided critical revisions of the the manuscript. All authors contributed to the article and approved the submitted version.

\section{FUNDING}

This work was supported by grants from the National Natural Science Foundation of China (No: 81671351 and 81801347) and the Planned Science and Technology Project of Guangdong Province, China (No: 2017B020227011). The funding organizations played no further role in study design, data collection, analysis and interpretation, and paper writing.

\section{ACKNOWLEDGMENTS}

We sincerely thank Yan Xia from the Department of Psychiatry, State University of New York Upstate Medical University for R statistical analyses. The authors thank the patients, volunteers, and their families whose participation made this work possible.

8. Bora E, Harrison BJ, Yucel M, Pantelis C. Cognitive impairment in euthymic major depressive disorder: a meta-analysis. Psychol Med. (2013) 43:2017-26. doi: 10.1017/S0033291712002085

9. Vinberg M, Miskowiak KW, Kessing LV. Impairment of executive function and attention predicts onset of affective disorder in healthy high-risk twins. $J$ Clin Psychiatry. (2013) 74:e747-53. doi: 10.4088/JCP.12m08258

10. Etkin A, Gyurak A, O'Hara R. A neurobiological approach to the cognitive deficits of psychiatric disorders. Dialogues Clin Neurosci. (2013) 15:419-29. doi: 10.31887/DCNS.2013.15.4/aetkin

11. Al-Fartusie FS, Al-Bairmani HK, Al-Garawi ZS, Yousif AH. Evaluation of some trace elements and vitamins in major depressive disorder patients: a case-control study. Biol Trace Elem Res. (2019) 189:412-9. doi: 10.1007/s12011-018-1507-7

12. Purchase R. The link between copper and Wilson's disease. Sci Prog. (2013) 96 (Pt 3):213-23. doi: 10.3184/003685013X13712193905878

13. Dening TR, Berrios GE. Wilson's disease. Psychiatric symptoms in 195 cases. Arch Gen Psychiatry. (1989) 46:1126-34. doi: 10.1001/archpsyc. 1989.01810120068011

14. Schlegel-Zawadzka M, Zieba A, Dudek D, Zak-Knapik J, Nowak G. Is serum copper a "trait marker" of unipolar depression? A preliminary clinical study. Pol J Pharmacol. (1999) 51:535-8.

15. Styczen K, Sowa-Kucma M, Siwek M, Dudek D, Reczynski W, Misztak P, et al. Study of the serum copper levels in patients with major depressive disorder. Biol Trace Elem Res. (2016) 174:287-93. doi: 10.1007/s12011-016-0720-5 
16. Nguyen SD, Kim JR, Kim MR, Jung TS, Soka DE. Copper ions and hypochlorite are mainly responsible for oxidative inactivation of paraoxonhydrolyzing activity in human high density lipoprotein. Toxicol Lett. (2004) 147:201-8. doi: 10.1016/j.toxlet.2003.12.011

17. Catena-Dell'Osso M, Bellantuono C, Consoli G, Baroni S, Rotella F, Marazziti D. Inflammatory and neurodegenerative pathways in depression: a new avenue for antidepressant development? Curr Med Chem. (2011) 18:245-55. doi: $10.2174 / 092986711794088353$

18. Fengler S, Roeske S, Heber I, Reetz K, Schulz JB, Riedel O, et al. Verbal memory declines more in female patients with Parkinson's disease: the importance of gender-corrected normative data. Psychol Med. (2016) 46:227586. doi: $10.1017 /$ S0033291716000908

19. Kim GH, Kim JE, Rhie SJ, Yoon S. The role of oxidative stress in neurodegenerative diseases. Exp Neurobiol. (2015) 24:325-40. doi: $10.5607 /$ en.2015.24.4.325

20. Drevets WC, Price JL, Furey ML. Brain structural and functional abnormalities in mood disorders: implications for neurocircuitry models of depression. Brain Struct Funct. (2008) 213:93-118. doi: 10.1007/s00429-008-0189-x

21. Jaworska N, Yang XR, Knott V, MacQueen G. A review of fMRI studies during visual emotive processing in major depressive disorder. World J Biol Psychiatry. (2015) 16:448-71. doi: 10.3109/15622975.2014.885659

22. Kempton MJ, Salvador Z, Munafo MR, Geddes JR, Simmons A, Frangou S, et al. Structural neuroimaging studies in major depressive disorder. Metaanalysis and comparison with bipolar disorder. Arch Gen Psychiatry. (2011) 68:675-90. doi: 10.1001/archgenpsychiatry.2011.60

23. Arsalidou M, Duerden EG, Taylor MJ. The centre of the brain: topographical model of motor, cognitive, affective, and somatosensory functions of the basal ganglia. Hum Brain Mapp. (2013) 34:3031-54. doi: 10.1002/hbm.22124

24. Loh WY, Anderson PJ, Cheong JLY, Spittle AJ, Chen J, Lee KJ, et al. Neonatal basal ganglia and thalamic volumes: very preterm birth and 7-year neurodevelopmental outcomes. Pediatr Res. (2017) 82:970-8. doi: $10.1038 /$ pr.2017.161

25. Shan Y, Jia Y, Zhong S, Li X, Zhao H, Chen J, et al. Correlations between working memory impairment and neurometabolites of prefrontal cortex and lenticular nucleus in patients with major depressive disorder. J Affect Disord. (2018) 227:236-42. doi: 10.1016/j.jad.2017.10.030

26. Hermens DF, Lagopoulos J, Naismith SL, Tobias-Webb J, Hickie IB. Distinct neurometabolic profiles are evident in the anterior cingulate of young people with major psychiatric disorders. Transl Psychiatry. (2012) 2:e110. doi: $10.1038 /$ tp.2012.35

27. Lener MS, Iosifescu DV. In pursuit of neuroimaging biomarkers to guide treatment selection in major depressive disorder: a review of the literature. Ann N Y Acad Sci. (2015) 1344:50-65. doi: 10.1111/nyas.12759

28. Lai S, Zhong S, Liao X, Wang Y, Huang J, Zhang S, et al. Biochemical abnormalities in basal ganglia and executive dysfunction in acute- and euthymic-episode patients with bipolar disorder: a proton magnetic resonance spectroscopy study. J Affect Disord. (2018) 225:108-16. doi: 10.1016/j.jad.2017.07.036

29. Yildiz-Yesiloglu A, Ankerst DP. Review of $1 \mathrm{H}$ magnetic resonance spectroscopy findings in major depressive disorder: a meta-analysis. Psychiatry Res. (2006) 147:1-25. doi: 10.1016/j.pscychresns.2005.12.004

30. Vythilingam M, Charles HC, Tupler LA, Blitchington T, Kelly L, Krishnan KR. Focal and lateralized subcortical abnormalities in unipolar major depressive disorder: an automated multivoxel proton magnetic resonance spectroscopy study. Biol Psychiatry. (2003) 54:744-50. doi: 10.1016/S0006-3223(02)01908-X

31. Gabbay V, Hess DA, Liu S, Babb JS, Klein RG, Gonen O. Lateralized caudate metabolic abnormalities in adolescent major depressive disorder: a proton MR spectroscopy study. Am J Psychiatry. (2007) 164:1881-9. doi: 10.1176/appi.ajp.2007.06122032

32. Mao N, Fang J, Xie H, Liu X, Jiang X, Wang G, et al. Correlation between neurochemical metabolism and memory function in adolescent patients with depression: a multi-voxel (1)H magnetic resonance spectroscopy study. Psychiatry Clin Neurosci. (2016) 70:167-74. doi: 10.1111/pcn.12372

33. Ende G, Braus DF, Walter S, Weber-Fahr W, Henn FA. Multiregional $1 \mathrm{H}-\mathrm{MRSI}$ of the hippocampus, thalamus, and basal ganglia in schizophrenia. Eur Arch Psychiatry Clin Neurosci. (2003) 253:9-15. doi: 10.1007/s00406-003-0398-5
34. Lundin F, Tisell A, Dahlqvist Leinhard O, Tullberg M, Wikkelso C, Lundberg $\mathrm{P}$, et al. Reduced thalamic $\mathrm{N}$-acetylaspartate in idiopathic normal pressure hydrocephalus: a controlled $1 \mathrm{H}$-magnetic resonance spectroscopy study of frontal deep white matter and the thalamus using absolute quantification. $J$ Neurol Neurosurg Psychiatry. (2011) 82:772-8. doi: 10.1136/jnnp.2010.223529

35. Zhong S, Wang Y, Lai S, Liu T, Liao X, Chen G, et al. Associations between executive function impairment and biochemical abnormalities in bipolar disorder with suicidal ideation. J Affect Disord. (2018) 241:282-90. doi: 10.1016/j.jad.2018.08.031

36. Abbaoui A, O ELH, Gamrani H. The neuronal basis of copper induced modulation of anxiety state in rat. Acta Histochem. (2017) 119:10-7. doi: 10.1016/j.acthis.2016.10.003

37. Liu X, Zhong S, Li Z, Chen J, Wang Y, Lai S, et al. Serum copper and zinc levels correlate with biochemical metabolite ratios in the prefrontal cortex and lentiform nucleus of patients with major depressive disorder. Prog Neuropsychopharmacol Biol Psychiatry. (2020) 99:109828. doi: 10.1016/j.pnpbp.2019.109828

38. Crayton JW, Walsh WJ. Elevated serum copper levels in women with a history of post-partum depression. J Trace Elem Med Biol. (2007) 21:17-21. doi: 10.1016/j.jtemb.2006.10.001

39. Russo AJ. Analysis of plasma zinc and copper concentration, and perceived symptoms, in individuals with depression, post zinc and anti-oxidant therapy. Nutr Metab Insights. (2011) 4:19-27. doi: 10.4137/NMI.S6760

40. Pulai S, Biswas A, Roy A, Guin DS, Pandit A, Gangopadhyay G, et al. Clinical features, MRI brain, and MRS abnormalities of drugnaive neurologic Wilson's disease. Neurol India. (2014) 62:153-8. doi: $10.4103 / 0028-3886.132349$

41. Nikolova YS, Iruku SP, Lin CW, Conley ED, Puralewski R, French B, et al. FRAS1-related extracellular matrix 3 (FREM3) single-nucleotide polymorphism effects on gene expression, amygdala reactivity and perceptual processing speed: an accelerated aging pathway of depression risk. Front Psychol. (2015) 6:1377. doi: 10.3389/fpsyg.2015.01377

42. Li DD, Zhang W, Wang ZY, Zhao P. Serum copper, zinc, and iron levels in patients with Alzheimer's disease: a meta-analysis of case-control studies. Front Aging Neurosci. (2017) 9:300. doi: 10.3389/fnagi.2017.00300

43. Lam PK, Kritz-Silverstein D, Barrett Connor E, Milne D, Nielsen F, Gamst $\mathrm{A}$, et al. Plasma trace elements and cognitive function in older men and women: the Rancho Bernardo study. J Nutr Health Aging. (2008) 12:22-27. doi: 10.1007/BF02982160

44. Travaglia A, La Mendola D, Magri A, Nicoletti VG, Pietropaolo A, Rizzarelli E. Copper, BDNF and its N-terminal domain: inorganic features and biological perspectives. Chemistry. (2012) 18:15618-31. doi: 10.1002/chem.201202775

45. Hwang JJ, Park MH, Koh JY. Copper activates TrkB in cortical neurons in a metalloproteinase-dependent manner. J Neurosci Res. (2007) 85:2160-6. doi: $10.1002 /$ jnr. 21350

46. Naletova I, Satriano C, Pietropaolo A, Giani F, Pandini G, Triaca V, et al. The copper(II)-assisted connection between NGF and BDNF by means of nerve growth factor-mimicking short peptides. Cells. (2019) 8:301. doi: $10.3390 /$ cells 8040301

47. Siwek M, Styczen K, Sowa-Kucma M, Dudek D, Reczynski W, Szewczyk B, et al. The serum concentration of copper in bipolar disorder. Psychiatr Pol. (2017) 51:469-81. doi: 10.12740/PP/OnlineFirst/65250

48. Wei C, Sun Y, Chen N, Chen S, Xiu M, Zhang X. Interaction of oxidative stress and BDNF on executive dysfunction in patients with chronic schizophrenia. Psychoneuroendocrinology. (2020) 111:104473. doi: 10.1016/j.psyneuen.2019.104473

49. Tsai G, Coyle JT. N-acetylaspartate in neuropsychiatric disorders. Prog Neurobiol. (1995) 46:531-40. doi: 10.1016/0301-0082(95) 00014-M

50. Ariyannur PS, Madhavarao CN, Namboodiri AM. N-acetylaspartate synthesis in the brain: mitochondria vs. microsomes. Brain Res. (2008) 1227:34-41. doi: 10.1016/j.brainres.2008.06.040

51. Xu H, Yang HJ, Rose GM, Li XM. Recovery of behavioral changes and compromised white matter in C57BL/6 mice exposed to cuprizone: effects of antipsychotic drugs. Front Behav Neurosci. (2011) 5:31. doi: 10.3389/fnbeh.2011.00031

52. Gregg JR, Herring NR, Naydenov AV, Hanlin RP, Konradi C. Downregulation of oligodendrocyte transcripts is associated with impaired 
prefrontal cortex function in rats. Schizophr Res. (2009) 113:277-87. doi: 10.1016/j.schres.2009.05.023

53. Murrough JW, Abdallah CG, Mathew SJ. Targeting glutamate signalling in depression: progress and prospects. Nat Rev Drug Discov. (2017) 16:472-86. doi: $10.1038 / \mathrm{nrd} .2017 .16$

54. Waddell KW, Zanjanipour P, Pradhan S, Xu L, Welch EB, Joers JM, et al. Anterior cingulate and cerebellar GABA and Glu correlations measured by (1)H J-difference spectroscopy. Magn Reson Imaging. (2011) 29:19-24. doi: 10.1016/j.mri.2010.07.005

55. Salustri C, Squitti R, Zappasodi F, Ventriglia M, Bevacqua MG, Fontana $\mathrm{M}$, et al. Oxidative stress and brain glutamate-mediated excitability in depressed patients. J Affect Disord. (2010) 127:321-5. doi: 10.1016/j.jad.2010. 05.012

56. Maes M, Vandoolaeghe E, Neels H, Demedts P, Wauters A, Meltzer HY, et al. Lower serum zinc in major depression is a sensitive marker of treatment resistance and of the immune/inflammatory response in that illness. Biol Psychiatry. (1997) 42:349-58. doi: 10.1016/S0006-3223(96)00 365-4

57. Nowak G. Zinc, future mono/adjunctive therapy for depression: mechanisms of antidepressant action. Pharmacol Rep. (2015) 67:659-62. doi: 10.1016/j.pharep.2015.01.015

58. Li Z, Wang G, Zhong S, Liao X, Lai S, Shan Y, et al. Alleviation of cognitive deficits and high copper levels by an NMDA receptor antagonist in a rat depression model. Compr Psychiatry. (2020) 102:152200. doi: 10.1016/j.comppsych.2020.152200

59. Slupski J, Cubala WJ, Gorska N, Slupska A, Galuszko-Wegielnik M. Copper concentrations in ketamine therapy for treatment-resistant depression. Brain Sci. (2020) 10:971. doi: 10.3390/brainsci101 20971

60. Haroon E, Fleischer CC, Felger JC, Chen X, Woolwine BJ, Patel T, et al. Conceptual convergence: increased inflammation is associated with increased basal ganglia glutamate in patients with major depression. Mol Psychiatry. (2016) 21:1351-7. doi: 10.1038/mp. 2015.206

61. Peters AT, Ren X, Bessette KL, Goldstein BI, West AE, Langenecker SA, et al. Interplay between pro-inflammatory cytokines, childhood trauma, and executive function in depressed adolescents. J Psychiatr Res. (2019) 114:1-10. doi: 10.1016/j.jpsychires.2019.03.030

62. Kaczmarczyk M, Wingenfeld K, Kuehl LK, Otte C, Hinkelmann K. Childhood trauma and diagnosis of major depression: association with memory and executive function. Psychiatry Res. (2018) 270:880-6. doi: 10.1016/j.psychres.2018.10.071

Conflict of Interest: The authors declare that the research was conducted in the absence of any commercial or financial relationships that could be construed as a potential conflict of interest.

Copyright (๑) 2021 Liao, Lai, Zhong, Wang, Zhang, Shen, Huang, Chen, Chen and Jia. This is an open-access article distributed under the terms of the Creative Commons Attribution License (CC BY). The use, distribution or reproduction in other forums is permitted, provided the original author(s) and the copyright owner(s) are credited and that the original publication in this journal is cited, in accordance with accepted academic practice. No use, distribution or reproduction is permitted which does not comply with these terms. 